Kalpa Publications in Engineering
Volume 3, 2020, Pages 212-222
Proceedings of International Sym-
posium on Applied Science 2019

\title{
Quantitative OCT Angiography of Retina Vessel Density for Early Glaucoma Diagnosis
}

\author{
Nhat Tan Le ${ }^{1}$, Tan Thi Pham ${ }^{1}$, Thanh Hoan Ngo ${ }^{2}$ \\ ${ }^{1}$ Faculty of Applied Science, Ho Chi Minh City University of Technology, Vietnam National \\ University Ho Chi Minh City, Vietnam \\ ${ }^{2}$ Department of Biomedical Engineering, International University, Vietnam National University \\ Ho Chi Minh City \\ lntan.sdh19@hcmut.edu.vn, ptthi@hcmut.edu.vn
}

\begin{abstract}
Glaucoma is the leading cause of irreversible blindness worldwide. Developed recently, OCTA is a promising non-invasive eyes imaging tool to glaucoma diagnosis in the early stage. This research designed a diagnosis support software based on analyzing color-density map and ROIs vessel density index on the OCTA images scanned peripapillary and macula area. Hessian-based filter and Otsu thresholding were used to detect and enhance small vessels. The program greatly detected areas of vascular dropout on glaucoma eyes.
\end{abstract}

\section{Introduction}

Glaucoma is one of the most common eyes disease over the world. The main causes of glaucoma come from a type of liquid called aqueous humor, which were contained by anterior and posterior chamber of the eyeballs. This fluid carries out nutrients transport processes between the eyeballs and the vein system of the body. In glaucoma eyes, this liquid was blocked or interrupted, stuck in the chamber that make the pressure inside the eye (intraocular pressure) increase by time and cause damage to the retina at the bottom of the eyeballs. This research was focusing on open-angle glaucoma, which more common cases in glaucoma.

Currently, glaucoma diagnosis methods were divided into two main groups: functional (Visual Field) and structural (eye's imaging) of the eyes. Both groups have their own strengths and limitations. Visual Field has been shown to have high diagnostic accuracy and that is the reason why Visual Field was the one of standard method for glaucoma diagnosis despite of long testing time (5 to 15 minutes) and the patient answers. For the diagnosis group based on structural changes in glaucoma such as measuring intraocular pressure (IOP) by Tonometry, measuring drainage angle by Gonioscopy and more commonly checking retinal lesions by a lot of eye's imaging methods such as ophthalmic funduscopy, optical coherence tomography (OCT) or especially optical coherence 
tomography angiography (OCTA). In OCTA, early diagnosis of glaucoma focus on images scans the macula, the optic nerve head (ONH) and the area of radial peripapillary capillary (RPC) [1].

Optical Coherence Tomography Angiography (OCTA) is a new eyes imaging method that can imaging the vessels systems of all the layers of the retina and choroid. With the abilities to detect minor damage in the retina, OCTA is the potential method for early glaucoma diagnosis according to recent studies. In OCTA, early diagnosis of glaucoma focus on images scans the macula, the optic nerve head $(\mathrm{ONH})$ and the area of radial peripapillary capillary (RPC) of the retina layers.

There are not a significant number of researches in the OCTA images scan the macula for glaucoma diagnosis but they found that vessel dropout in parafoveal of the macula can determine glaucomatous eyes. The analysis in this area focus on 2 layers: superficial plexus and deep plexus. In superficial plexus layer, diagnosis factors mainly were vascular density parameters [2]; In deep plexus used vascular density parameters [3] and size of the fovea avascular zone (FAZ) area [4]. Researches in FAZ area focused on the increase of area circumference and the reduction of the circular index (circularity index: the ratio of the inner tangent area and the entire area of FAZ) in glaucomatous eyes. In particular, the study [4] determined that the circular index FAZ could be a new factor showing disruption of the parafoveal capillary network in glaucoma. For blood vessels density parameters, many studies have shown decrease in vessel density in both layers, but the superficial layer has a significant reduction over deep plexus [5, 6, 7].

Researches in the OCTA images scanned the disc almost focused on vessel density dropout in $\mathrm{ONH}$ and especially RPC areas, the region were believed to have marked symptoms for early diagnosis of open-angle glaucoma (OAG). The first studies were conducted by Jia [8] focused directly on $\mathrm{ONH}$ area, demonstrated a reduction in vessel density in glaucomatous eyes but did not have any results in early diagnosis because of the big affection of major blood vessels. Major blood vessels do not have any changes in glaucomatous retina and these shadow obscure the capillary network in deeper layer. Later studies turned to the area around the disc (RPC) and showed that the diagnostic accuracy were comparable to current standard methods such as retinal nerve fiber layer thickness measurement on OCT and visual field testing $[5,6,9]$. That is the reason why vascular density in the $\mathrm{RPC}$ area is the most potential parameter for early diagnosis of glaucoma.

This research focused in the image scan the RPC and macula area for glaucoma diagnosis.

\section{Methods}

This research built a user interface based on Matlab Guide platform. The input is OCTA images and the outputs were color vessels density map and the vessels density index of regions of interest. Then program was tested on 2 OCTA image datasets (macula scan and RPC scan) to evaluate the accuracy of the program and the ability to glaucoma diagnosis in the early stage.

The idea of image processing algorithm is described through the diagram below (Figure 1). First, the imported OCTA image will be processed to obtain the major blood vessels map and the entire vessel binary image on the imported image. The major blood vessels map will be determined by using the Adaptive Threshold method. The retinal vascular binary image will be identified after two steps: enhance vascular structures by using Hessian-based filter and then segment by using the Otsu's threshold method. Micro-vessels binary image was determined by subtracting major blood vessels from the blood vessels binary image (two images just obtained in the previous step). The color vessel density map will be built by computing based on small windows and the vascular density parameters was calculated based on masks. 


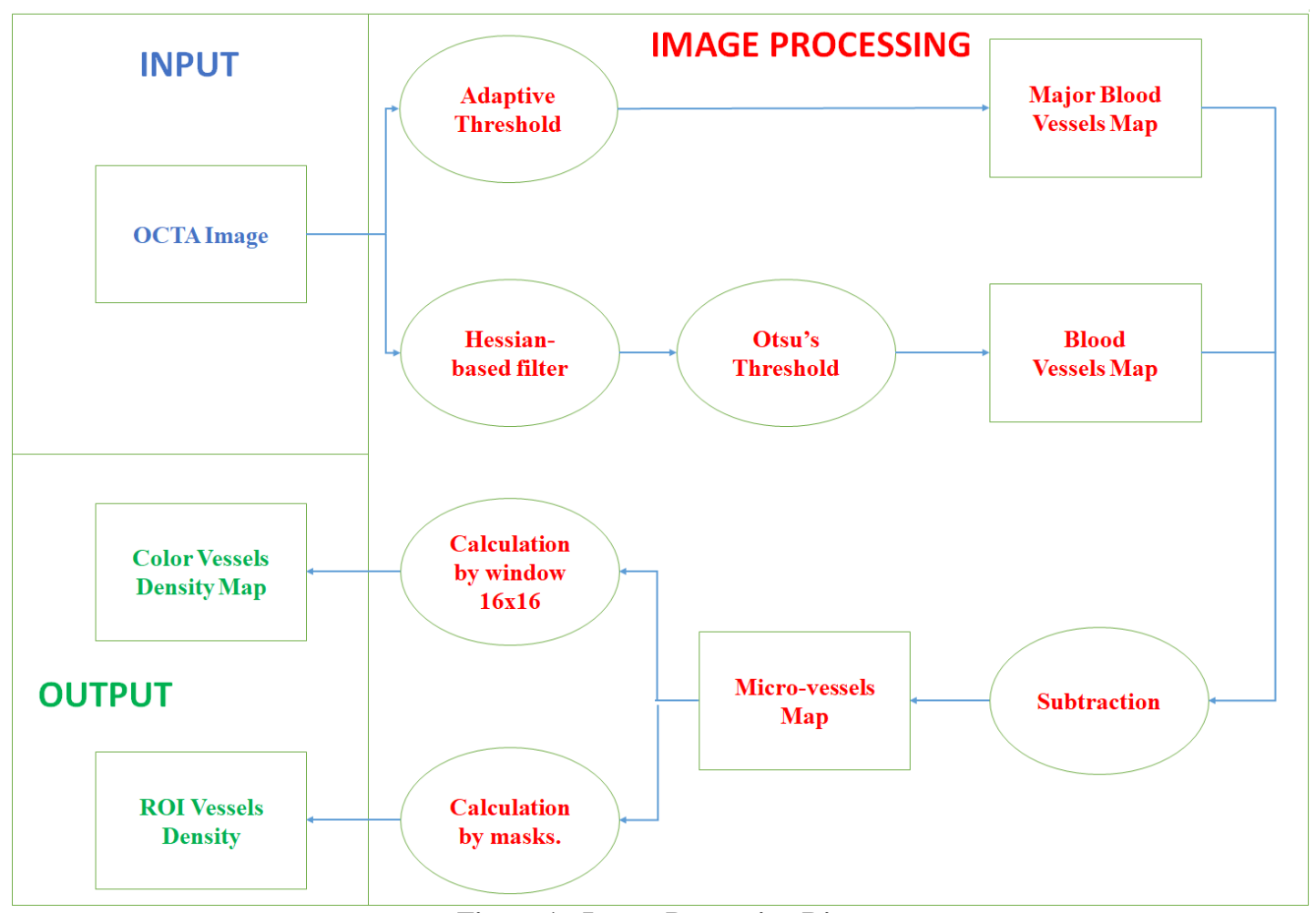

Figure 1 : Image Processing Diagram

\subsection{Determine major blood vessel image by using Adaptive Threshold}

First, the imported image was resized by the factor of 6 by using linear interpolation method to make it larger and easier to process. For example, the OCTA image scans the RPC area with a size of $4.5 \times 4.5 \mathrm{~mm}$ corresponding to a matrix of $304 \times 304$ pixels, after resizing, the matrix size will be 1824 x 1824 pixels.

Different from the single threshold, the Adaptive Threshold have different threshold values for each region. Determine local thresholds is based on the average of the pixel value in each area, and thereby calculating the automatic threshold for each region and pixel.

\subsection{Determine blood vessels binary images by combining Hessian- based Filter and Otsu's Threshold}

The image pre-processing method based on the Hessian-based filter is a vascular enhanced method first developed by Franji and his colleagues in 1998 and it is commonly used in vascular imaging problems such as DSA, CTA or MRA [10]. The idea of this algorithm is the structure of blood vessels and the pixel value distribution in the image. Although these structures have different gray levels and size, but almost of blood vessels are the tubular structures. Franji proved that the engine-values and engine-vectors of the Hessian matrix are closely related to the distribution of density and orientation of blood vessels in space. Based on these factors we can identify and enhance vascular structures on OCTA images. This program used Hessian-based filter developed by Jerman [11] in 2016, based on Franji's platform. 
Images segmentation to distinguish between blood vessels structures and background is very important for calculating vessels density and building the color density map. Otsu thresholding is a method highly effective use for image segmentation between two objects [12]. These two regions will divide based on the histogram. After using Hessian-based filter, histogram of the image was almost divided into two distinct areas. This method is based on calculating the threshold so that the total variance of the two regions is the smallest.

This program is an experiment when using the combination of vascular enhancement by Hessianbased filter and image segmentation by Otsu's threshold on OCTA image and gives better results compared to using only the segmentation with Otsu as shown in Figure 2C. Micro-vessels binary images will be determined by subtracting the two results obtained above (Figure 2D).

\subsection{Color vessel density map}

The color vessel density map was built by scanning and calculating with a window of $16 \times 16$ pixels across the entire micro-vessels binary image (Figure 2D). The local vessel density is calculated by dividing the ratio of white pixels to the total number of pixels of the window (256 pixels) and it is this scaled value that is assigned to all pixels on the density image. This density image will be color coded in the jet color of Matlab, with changes from cool colors to hot colors corresponding to the gradual increase density (Figure 2E). Therefore, areas of reduced density are easily identified by the area of cool colors (green, blue, ...) and commonly appear in early open-angle glaucoma cases.

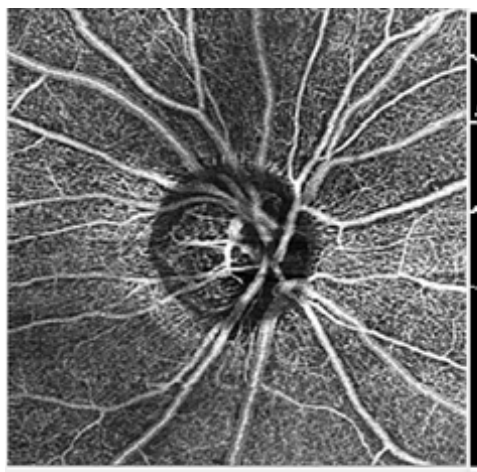

A

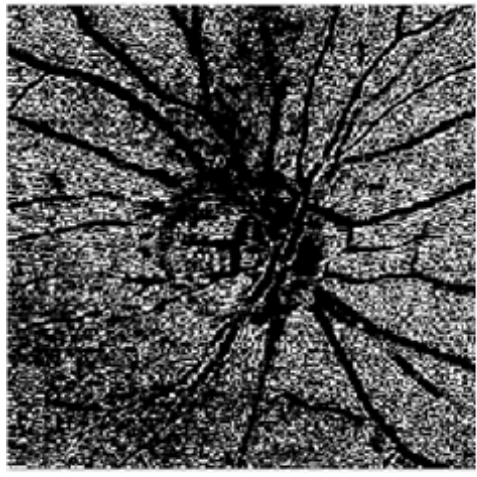

D

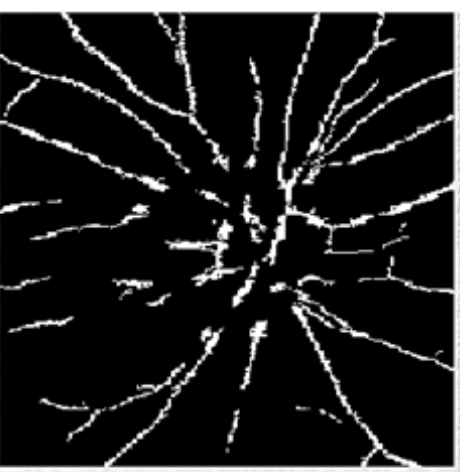

B

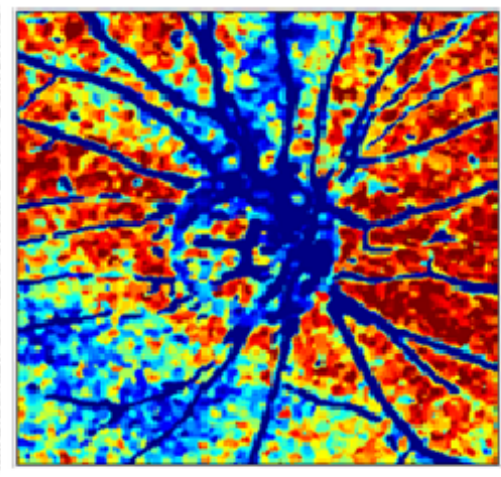

E

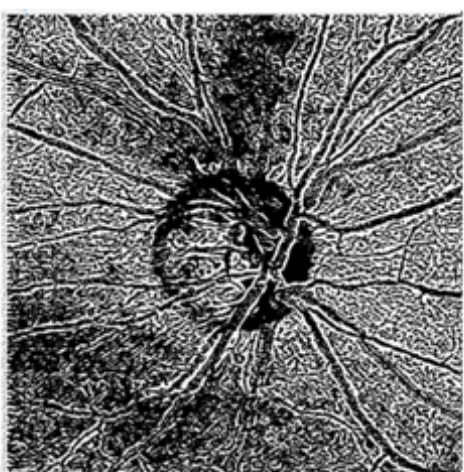

C

Figure 2: The original image (A); Results after processing: Major blood vessel image (B); Blood vessels binary image (C); Micro-vessels map (D); Color density map (E). 


\subsection{Calculate ROIs vessels density}

ROIs vessels density will be calculated similarly to the calculation for color map. ROIs were identified by masks to isolate each region and calculate vascular density in each area to determine early signs of glaucoma. The ROIs masks included: the disc area $(\mathrm{ONH})$, the annulus area around the disc was divided into 4 equal areas: Inferior, Nasal, Superior and Temporal (Figure 3). This annulus has an internal diameter of $1.95 \mathrm{~mm}$ and an outer diameter of $3.45 \mathrm{~mm}$. All the masks were designed and edited on ImageJ software. The vascular density parameter will be calculated on the seven areas of interest, the whole image, the disk $(\mathrm{ONH})$, the whole annulus and four areas in the annulus. This calculation provided additional information about vascular density of each ROI, supplement and support for diagnosis based on color vessels density maps.

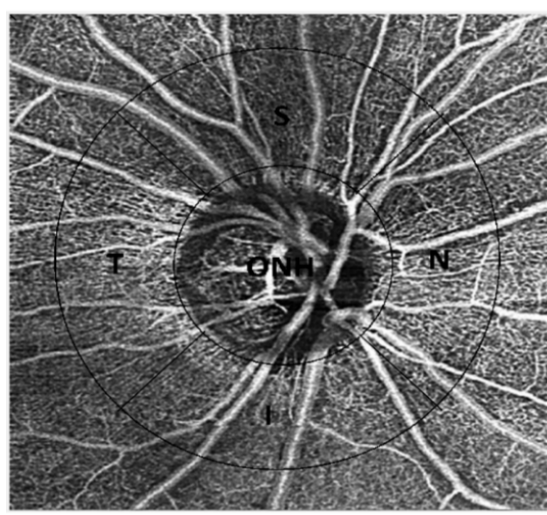

A
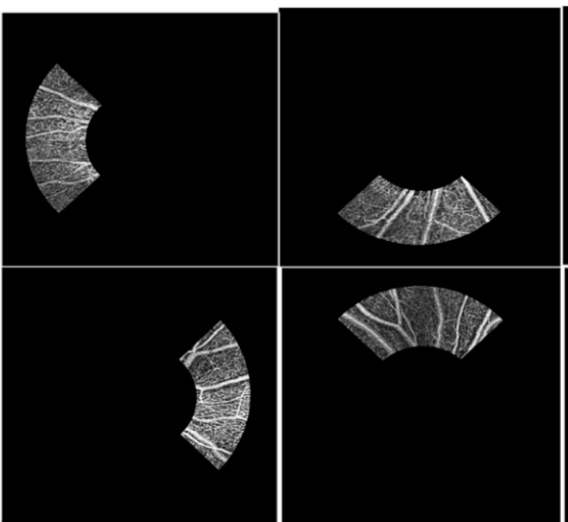

B

Figure 3: Seven ROI for blood vessel density in 4,5 x 4,5 mm OCTA image scan the disc (A). Six regions separated from the original image from the left to the top are Temporal, Inferior, whole annulus and bottom row are Nasal, Superior, ONH (B).

\subsection{Build an interface based on Matlab platform}

With the purpose of assisting doctors in glaucoma diagnosis, designing an easy-to-use interface is one of the goals of this research. The interface displayed result images and the vessels density of 7 regions of interest: whole-image, optic nerve head $(\mathrm{ONH})$, whole-annulus and 4 small areas in annulus (Superior, Nasal, Inferior, Temporal). (Figure 4) 

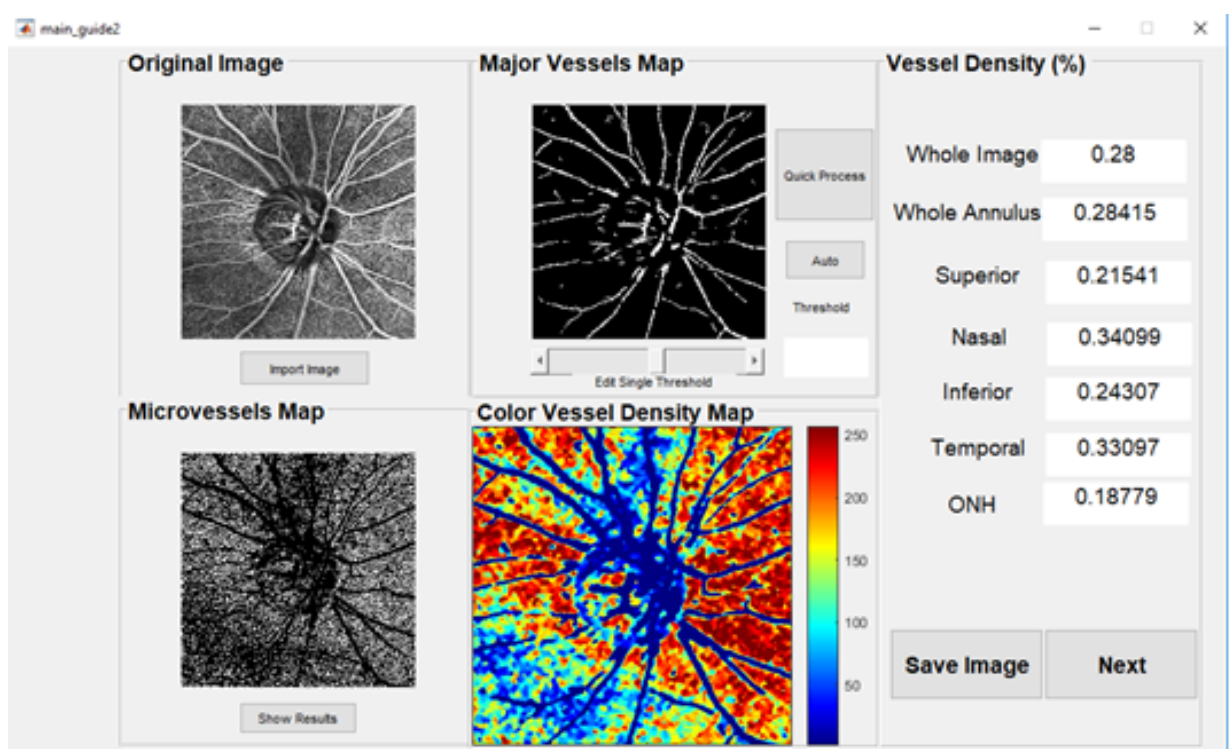

Figure 4: User Interface.

\subsection{Testing OCTA image databases}

Data set $1(\mathrm{n}=21): 3 \times 3 \mathrm{~mm}$ macula scan OCTA image of random healthy eyes. The program identified vessels color map and calculated the vessel density of the parafoveal region which divided into four regions (Figure 5A). To evaluate the accuracy of the program This index will be compared with the results of Choi's research [4] which analyzed on the same data.

Data set $2(\mathrm{n}=3): 4.5 \times 4.5 \mathrm{~mm}$ peripapillary scan OCTA image data of healthy, suspected glaucoma and glaucoma eyes. The program identified vessels color map and calculated the vessels density of the RPC, ONH and four small parts of RPC area (Figure 5B). The vessels density index of three cases were compared to give conclusion about the relationship between glaucoma process and vessels density.

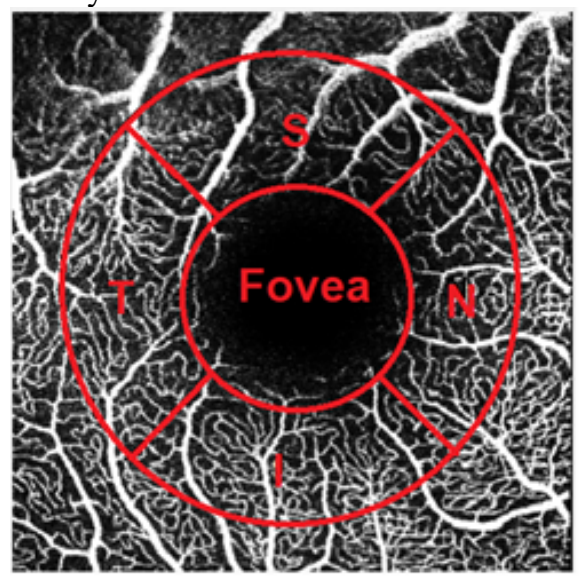

A
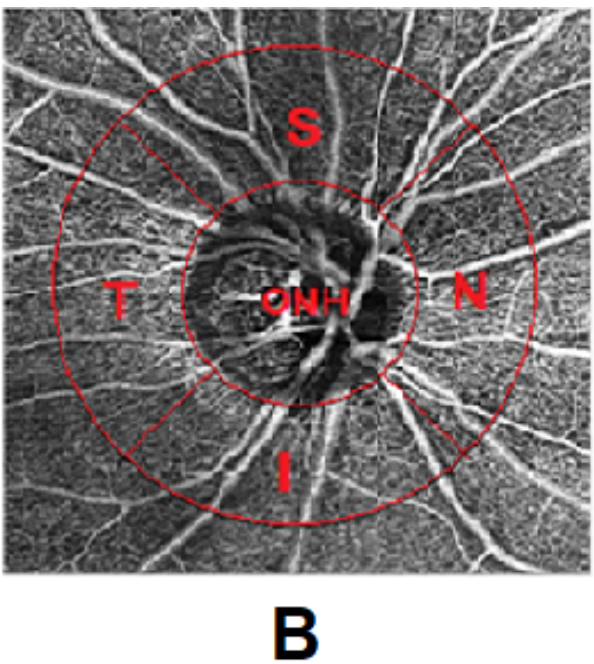

Figure 5: ROIs in macular scans (A) and RPC scans (B). 


\section{Results}

The results of dataset 1 were showed in Figure 6 (color vessels density map) and Table 1 (vessels density index). Color vessels density maps were mainly in the hot color because of imaging the healthy eyes. There were no significant differences in vascular density of each ROIs between the program and Choi's program results with the same type of OCTA image data, the largest difference was in the Temporal area (6.42\%).
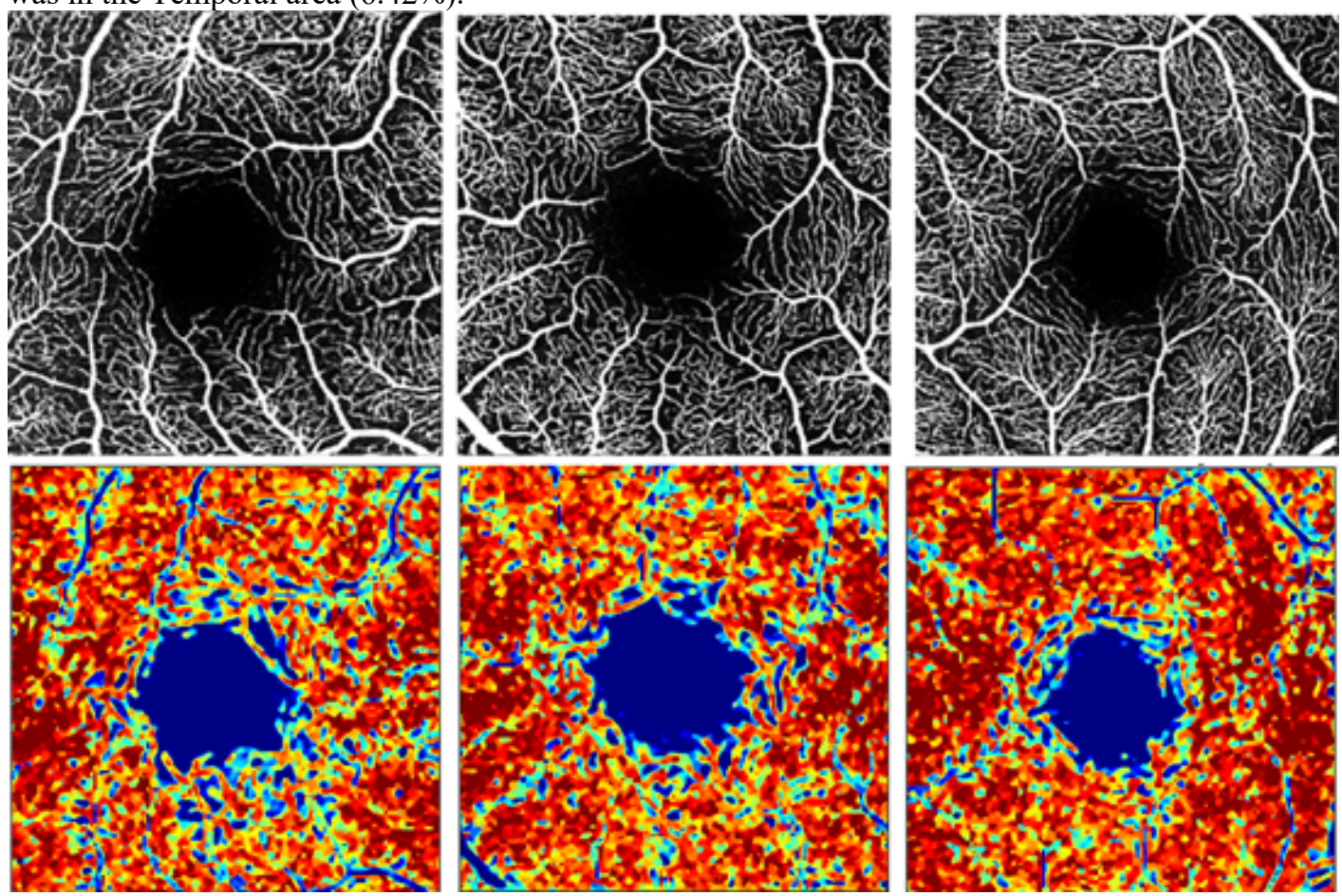

Figure 6: Color vessels density maps of OCTA image scan the macula data of healthy eyes.

\begin{tabular}{|c|c|c|}
\hline & $\begin{array}{c}\text { Vessels Density (\%) } \\
(\boldsymbol{n}=\mathbf{2 1})\end{array}$ & $\begin{array}{c}\text { Vessels Density (\%) } \\
(\text { Choi }[\mathbf{4}], \boldsymbol{n}=\mathbf{5 2})\end{array}$ \\
\hline Whole Image & $32.90 \pm 1.82$ & $30.08 \pm 6.83$ \\
\hline Parafoveal & $34.81 \pm 2.30$ & $30.20 \pm 7.61$ \\
\hline Superior & $35.81 \pm 4.95$ & $30.41 \pm 8.09$ \\
\hline Nasal & $32.62 \pm 4.23$ & $30.08 \pm 7.88$ \\
\hline Inferior & $33.57 \pm 3.76$ & $29.53 \pm 7.05$ \\
\hline Temporal & $37.20 \pm 5.27$ & $30.78 \pm 7.41$ \\
\hline Fovea & $11.91 \pm 3.90$ & \\
\hline
\end{tabular}

Table 1: The vessels density index of OCTA image scan the macula of healthy eyes data $(n=21)$, compared with the results of the study [4]. 
The results of dataset 1 were showed in Figure 7 (color vessels density map) and Table 2 (vessels density index). Vessels density index in glaucoma eyes had a significant decrease compared to healthy eyes: The vessels dropout density of the whole image was 7\% and for the RPC region was $7.9 \%$. The ROIs with the highest decrease were Superior (16.8\%), Inferior $(10.2 \%)$ and ONH (10.6\%). The Nasal (1.1\%) and Temporal (3.1\%) areas did not show significant decreases. Between the healthy eyes and the glaucoma suspected eyes did not have any significant changes, the highest decrease was in the Superior area with 3.6\% vessels density dropout and could be recognized on the vascular color map.

\section{Control eyes}

\section{Glaucoma Suspect}

\section{Glaucoma}
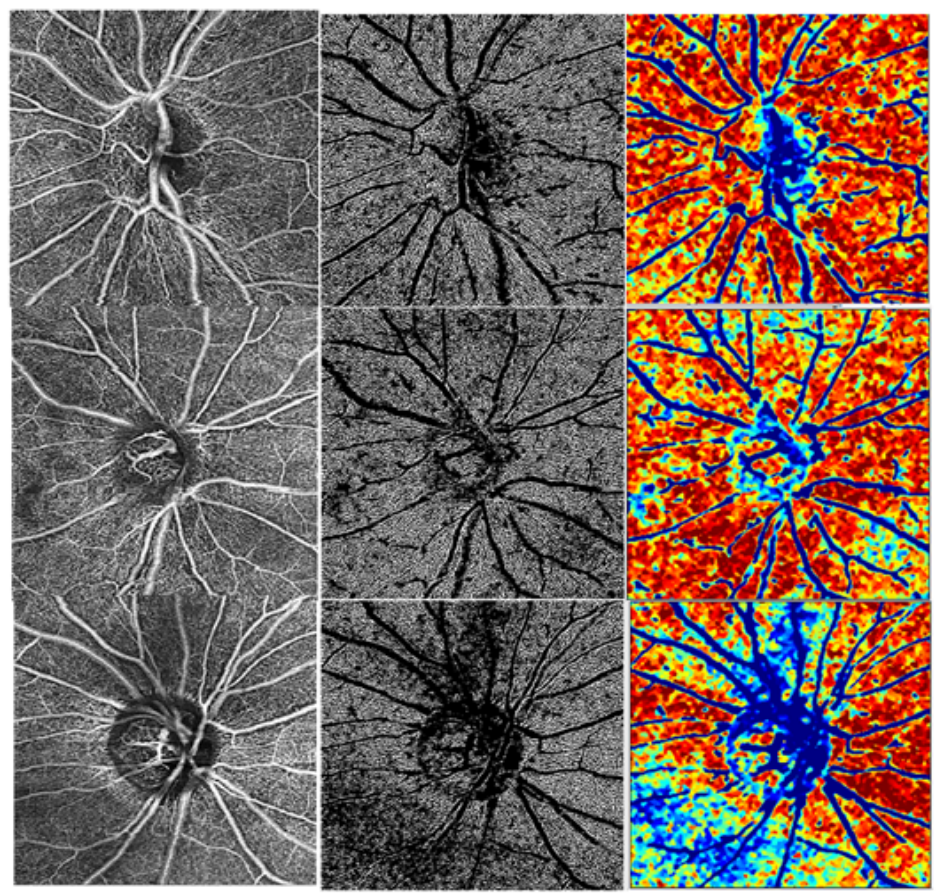

Figure 7: Color vessels density map of healthy, suspected glaucoma and glaucoma eyes. From the left is the original image, the major vessels subtraction binary image and color vessels density map. Regions with vessels dropout were easily recognized in glaucoma eyes.

\begin{tabular}{|c|c|c|c|}
\hline & \multicolumn{3}{|c|}{ Mật độ mạch máu (\%) } \\
\hline Whole Image & Control eyes & Glaucoma Suspect & Glaucoma \\
\hline Whole Anulus & 35 & 34 & 28 \\
\hline Superior & 36.3 & 35.3 & 28.4 \\
\hline Nasal & 38.3 & 34.7 & 34.1 \\
\hline Inferior & 35.2 & 33.8 & 24.3 \\
\hline Temporal & 34.5 & 36.0 & 33.1 \\
\hline ONH & 36.2 & 36.0 & 18.8 \\
\hline
\end{tabular}

Table 2: The vessels density index of OCTA image scan the RPC data $(n=21)$. 


\section{Discussion}

The density results on OCTA data scanned the macula area of 21 normal random healthy eyes gave a conclusion that this parameter was not significant affected by factors of humans like physical condition, gender, ... This is very important for data analysis process to give correctly clinical diagnosis of glaucoma by using this parameter. The results compared with another program on the same type of OCTA images showed that the accuracy of the program was acceptable.

The density results of OCTA data scanned the RPC of three cases have shown that blood vessel density parameters were an important key for glaucoma diagnosis. Lesions of glaucoma eyes in early stage were usually small areas, and often appear in the Superior region of the RPC area. The results of this research showed similar results when the small vessels dropout areas in the Superior area of images suspected to have glaucoma can be observed on the color vessels density map, even based on density parameters did not have significant decrease. For patients with glaucoma, significant vessels dropout can be clearly identified in Superior and Inferior areas.

Using Hessian-based filter as a vascular enhancement filter and Otsu threshold as an image segmentation method showed positive effects in enhance and fully identify the small vascular map. Hessian-based filter has been shown to deliver positive results in 3D MRA, DSA, CTA images for peripheral vascular imaging, thoracic applications and fluorescence image of the eyes [11]. This research had tested this filter to enhance a retinal vascular system shown in the OCTA image and recognized that vascular structures are mostly detected and strengthened, so that the Otsu threshold segmentation process was operated more effectively. Comparison between direct segmentation with Otsu and segmentation after using the Hessian-based filter has clearly shown this effectiveness.

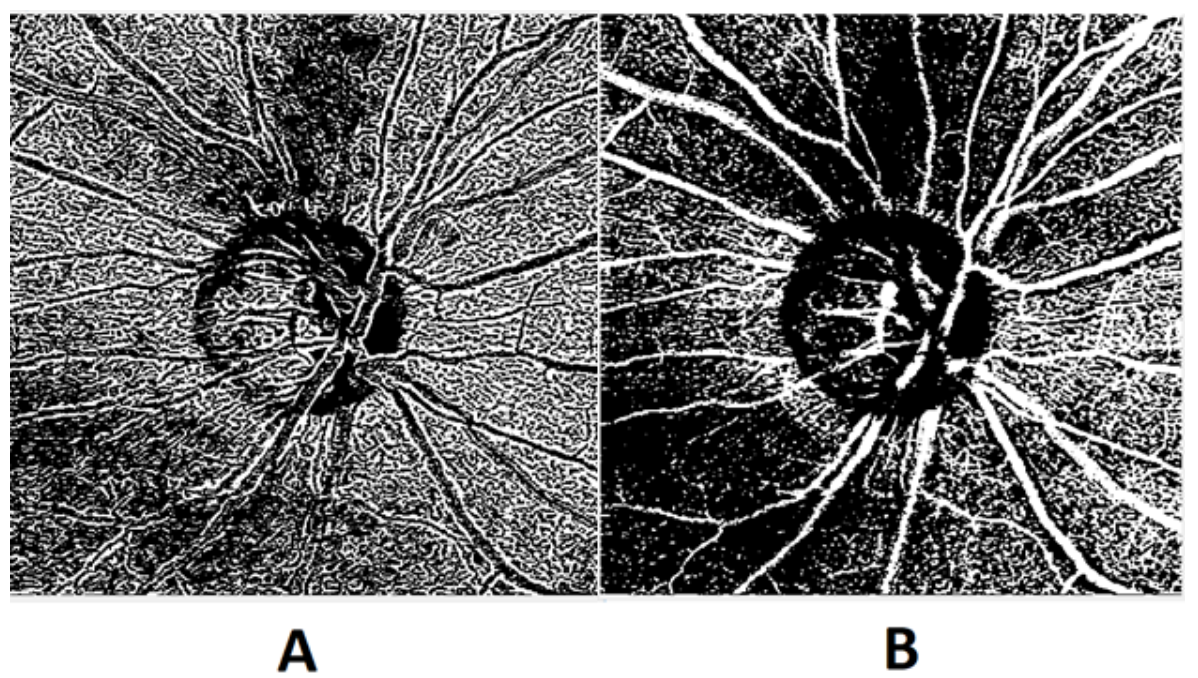

Figure 8: Results of micro-vessels map between using Otsu threshold and Hessian-based filter (A) and only Otsu threshold (B).

The user interface was designed give a fast processing tools to support ophthalmology doctor have a better clinical decision. Allowing user to import OCTA images and receive output vascular density results as well as vascular color maps. Display all the results step-by-step depending on the user's requirements. This interface will be the basis for further development to produce more meaningful diagnostic results.

However, this research also had limitations. Firstly, the OCTA image dataset scanned the RPC area was limited, not enough give any deeply conclusions about the method's ability to detect 
glaucoma. Secondly, running the program on the OCTA image data set scans the macula area only in healthy eye samples. Thirdly there was no standard program to compare and evaluate the program more accurately. However, in the future this program would have to test on a lot of databases. In addition to proving the effectiveness of using the vascular density parameter, more research in data analysis to provide more diagnostic results is needed.

\section{Conclusions}

The research has successfully built the program and user interface to identify color vessels density maps and calculate the vessels density parameters of the ROIs on the OCTA image scanning the macula and the RPC area to supporting early open-angle glaucoma diagnosis. The reliability of the program was good when compared to other programs running on the same data type. The program also showed similar results when studying on OCTA image of the glaucoma eyes. Algorithms with improvements in enhancement and segmentation of blood vessels give positive results. The program interface was an easy-to-use tool and will be developed to helping ophthalmologists in early glaucoma diagnose in the future.

\section{References}

[1] Van Melkebeke L., Barbosa-Breda J., Huygens M., Stalmans I, "Optical Coherence Tomography Angiography in Glaucoma: A Review," Ophthalmic Research, vol. 60, pp. 139$151,2018$.

[2] Henry Shen-Lih Chen, Chun-Hsiu Liu, Wei-Chi Wu, Hsiao-Jung Tseng, Yung-Sung Lee, "Optical Coherence Tomography Angiography of the Superficial Microvasculature in the Macular and Peripapillary Areas in Glaucomatous and Healthy Eyes," Investigative Ophthalmology \& Visual Science, vol. 58, p. 3637-3645, 2017.

[3] Min Hee Suh, Linda M. Zangwill, Patricia Isabel C. Manalastas, Akram Belghith, Adeleh Yarmohammadi, Felipe A. Medeiros, Alberto Diniz-Filho, Luke J. Saunders, Robert N. Weinreb, "Deep Retinal Layer Microvasculature Dropout Detected by the Optical Coherence Tomography Angiography in Glaucoma," Ophthalmology, vol. 123, no. 12, pp. 2509-2518, 2016.

[4] Jaewan Choi, Junki Kwon, Joong Won Shin, Jiyun Lee, Saem Lee, Michael S. Kook, "Quantitative optical coherence tomography angiography of macular vascular structure and foveal avascular zone in glaucoma," PLOS ONE, vol. 12, p. e0184948, 2017.

[5] Handan Akil, Alex S. Huang, Brian A. Francis, Sirinivas R. Sadda, "Retinal vessel density from optical coherence tomography angiography to differentiate early glaucoma, pre-perimetric glaucoma and normal eyes," PLOS ONE, vol. 12, p. e0170476, 2017.

[6] Maged Alnawaiseh, Larissa Lahme, Viktoria Müller, André Rosentreter, Nicole Eter, "Correlation of flow density, as measured using optical coherence tomography angiography, with structural and functional parameters in glaucoma patients," Graefes Arch Clin Exp Ophthalmol, vol. 256, pp. 589-597, 2018.

[7] Richard F. Spaide, Christine A. Curcio, "Deep Vascular Layers of the Retina by Optical Coherence Tomography Angiography Instruments in Normal Eyes," JAMA Ophthalmol, vol. 135, pp. 259-262, 2017. 
[8] Yali Jia, John C. Morrison, Jason Tokayer, Ou Tan, Lorinna Lombardi, Bernhard Baumann, Chen D. Lu, WooJhon Choi, James G. Fujimoto, and David Huang, "Quantitative OCT angiography of optic nerve head blood flow," Biomed Opt Express, vol. 3, no. 12, p. 31273137, 2012.

[9] Lawrence S Geyman, Reena A Garg, Yanin Suwan, Vivek Trivedi, Brian D Krawitz, Shelley Mo, Alexander Pinhas, Apichat Tantraworasin, Toco Y P Chui, Robert Ritch, Richard B Rosen, "Peripapillary perfused capillary density in primary open - angle glaucoma across disease stage : an optical coherence tomography angiography study," British Journal of Ophthalmology, vol. 101, 2017.

[10] Alejandro F. Frangi, Wiro J. Niessen, Koen L. Vincken, Max A. Viergever, "Multiscale vessel enhancement filtering," Proc. Med. Image Comput. Assist. Interv., vol. 1496, p. 130-137, 1998.

[11] Tim Jerman, Franjo Pernus, Bo stjan Likar, and Ziga Spiclin, "Enhancement of Vascular Structures in 3D and 2D Angiographic Images," IEEE Transactions on Medical Imaging, vol. 35, no. 9, pp. 2107 - 2118, 2016.

[12] Hui-FuangNg, "Automatic thresholding for defect detection," Pattern Recognition Letters, vol. 27, no. 14, pp. 1644-1649, 2006. 